\title{
Networking in the visual cortex
}

The organisation of synaptic wiring in the brain is fundamental to its many functions. The dense and overlapping local synaptic connectivity of neurons in the sensory cortex is not random, suggesting that the connectivity pattern has a role in the processing that occurs in this brain area; however, little is known about the rules determining which cells are interconnected. In a new paper, Ko, Hofer et al. reveal that, in the mouse visual cortex, cells that share functional properties are more likely to be synaptically connected.

To examine both the functional properties and synaptic connectivity of individual neurons, the authors

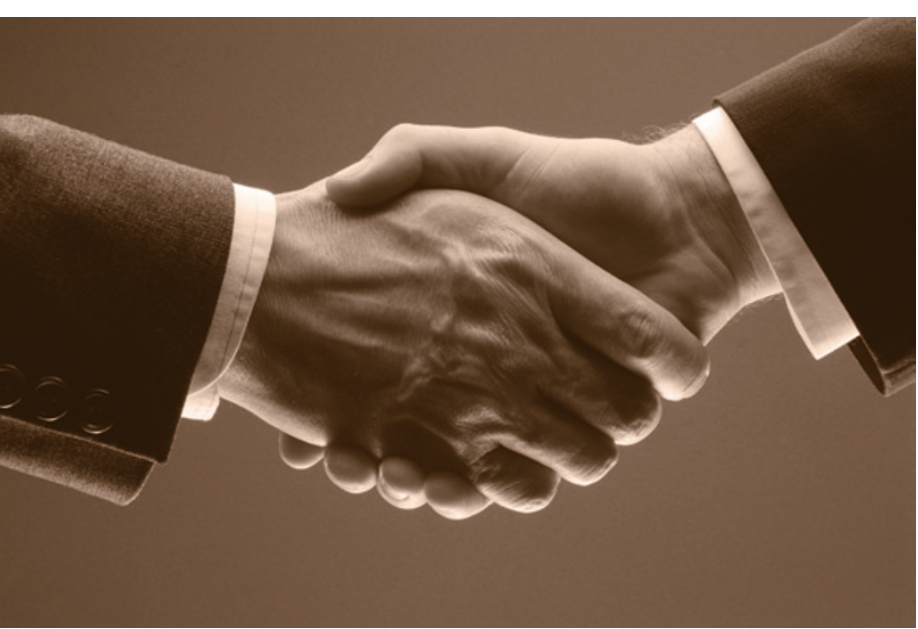

first used in vivo two-photon calcium imaging to map the responses of all the pyramidal neurons in a small area of layer 2-3 of the mouse visual cortex to two types of stimuli: drifting gratings and natural movie sequences. Next, they took slices of this brain region and performed patch-clamp recordings in up to four neighbouring neurons at a time. By evoking action potentials in one cell and recording from the other three, the authors determined whether these neurons were synaptically connected. Finally, they precisely matched the functional and electrophysiological data for individual cells through use of computer software that aligned anatomical image stacks captured during each part of the study.

By obtaining both in vivo and in vitro recordings from 116 neurons in total, the authors made a number of important discoveries. Neurons that responded to moving gratings were more highly connected to each other than to neurons that did not respond to such stimuli. Moreover, neurons that fired preferentially in response to a particular orientation of the moving grating were also more likely to connect to other neurons with the same orientation preference. Interestingly, the direction selectivity of the neurons did not seem to be related to their interconnectivity.
The authors found similar results with natural movie sequences: neurons that responded to such stimuli were more likely to be synaptically connected to each other than to those cells that did not respond. Connected neurons also showed similar variability in neuronal firing during repeated presentation of the same stimulus. Moreover, the number of bidirectional neuronal connections between pairs of neurons with similar response properties (to either moving gratings or natural movies) was higher than between those with properties that differed.

These findings show that although neurons in visual processing networks are widely interconnected, there is a bias towards connectivity with cells that share certain functional properties. Issues that need to be addressed include how this connectivity pattern arises during development and how these local networks contribute to the overall processing of visual stimuli. Using this method in other optically accessible brain regions may improve our general understanding of information processing in complex neural circuits.

Katherine Whalley

ORIGINAL RESEARCH PAPER Ko, H. et al. Functional specificity of local synaptic

connections in neocortical networks. Nature 10 Apr 2011 (doi:10.1038/nature09880) 